\title{
Classification Of Faults During Integration Of Hybrid System To Microgrid Using Neuro-Fuzzy Technique
}

\author{
Ritu Singh $^{a}$, Soorya Prakash Shukla ${ }^{b}$, Subhendu Patic ${ }^{c}$ P.R. Thakurad \\ aDepartment of Electrical Engineering, CSVTU, Bhilai, India. E-mail: ritusingh02ee@ gmail.com \\ bDepartment of Electrical Engineering, BIT, Durg, India. E-mail: sp.shukla@bitdurg.ac.in \\ 'Department of Electrical Engineering, Siksha O Anusnadhan University, Bhubaneswar, India. \\ E-mail: subhendupati@soa.ac.in \\ ${ }^{\mathrm{d}}$ Department of Electrical and Electronics Engineering, BIT Mesra, Ranchi, India. E-mail: \\ prthakura@bitmesra.ac.in
}

Article History: Received: 11 January 2021; Accepted: 27 February 2021; Published online: 5 April 2021

\begin{abstract}
To improve microgrid efficiency, detecting the fault as soon as possible is imperative. Taking into account the above problem a peculiar technique has been implemented in the analysis of this paper with a micro grid consisting of wind turbine (WT) and diesel generator for the classification of different types of faults. All Neuro-fuzzy (NF) fault disturbances are introduced by taking the input function data for accurate classification of different faults. One technically applicable 3-bus framework integrated with various forms of delivery generations that are considered for the purpose of security analysis and the simulation is done for the environment using MATLAB / SIMULINK. The fault classification is achieved by using Neuro Fuzzy. The results of the comparison are presented, showing the improved performance of Neuro Fuzzy (NF).
\end{abstract}

Keywords: Microgrid, Fuzzy logic (FL), Artificial Neural Network (ANN), Neuro Fuzzy (NF).

\section{Introduction}

Hybrid system is the combination of renewable (wind) as well as conventional (diesel) energy system. The wind turbine system and the diesel system provide the continuity of supply even in the faulted condition. Although the uninterrupted power supply is maintained, but for the safety of power system, it is necessary to detect the faults so as protect the transmission lines. The faults in the lines are normally caused due to several reasons like human mistakes and bad weather conditions, etc. These faults can be classed into two types. One is open conductor faults or series faults and another one is short circuit faults or shunt faults. Shunt faults are classified as symmetrical and unsymmetrical faults. Again symmetrical faults are LLL, LLLG faults whereas asymmetrical faults are LG, LL, and LLG etc.

Islanding refers to a scenario where a part of the electrical power system such as generator and load is isolated from the electrical grid or source but system still remains energized. It becomes important wherein the installed generators are coordinated at conveyance level. It is thus desired to have islanding in large systems, because if any severe disturbance occurs then instead of collapsing of the entire system it is better to have islanding. Islanding has got an advantage that whenever there is a severe disturbance in the system the entire framework isn't lost and its rebuilding is straightforward.

Many techniques have been developed for the classification of faults. However, this paper makes use of one of the hybrid technique called Neuro-fuzzy technique, which integrates the artificial neural network (ANN) and fuzzy logic (FL). ANN is an intelligent algorithm which is capable of identifying the system behavior, whereas FL is capable of controlling the variable parameters of the system [1]. Fuzzy logic is basically a rule based approach which makes use of the IF-TEHN relation [4]. The hybrid technique adds the training ability of ANN to rule based fuzzy system which makes it viable to learn from the previously obtained data sets [6].

As a well-known representative of all training algorithms, back propagation technique is widely used in neural network which is discussed below.

Following the introduction this paper describes the Neuro-fuzzy technique [8] in section II, then its proposed model in section III followed by the results in section IV. The paper concluded in the last section.

\section{Neuro-Fuzzy Technique}

One of the ingenious algorithms that is used for recognition and control is the artificial neural network. Neural networks can assimilate the actions of the system. These networks can be used to recognize the relevant 
system behavior in non-linear system [2]. Fuzzy logic is a propitious instrument in the control engineering, which helps to control the parameters in the real time scenario [3]. Incorporation of fuzzy logic in artificial neural network leads to fruitful and valuable output. Artificial neural network can be trained, but no such training is possible in fuzzy logic [2].

By integrating these two techniques, creates a new hybrid technique known as adaptive neuro-fuzzy inference system (ANFIS) which helps in memorizing the data set by providing an adaptive learning procedure

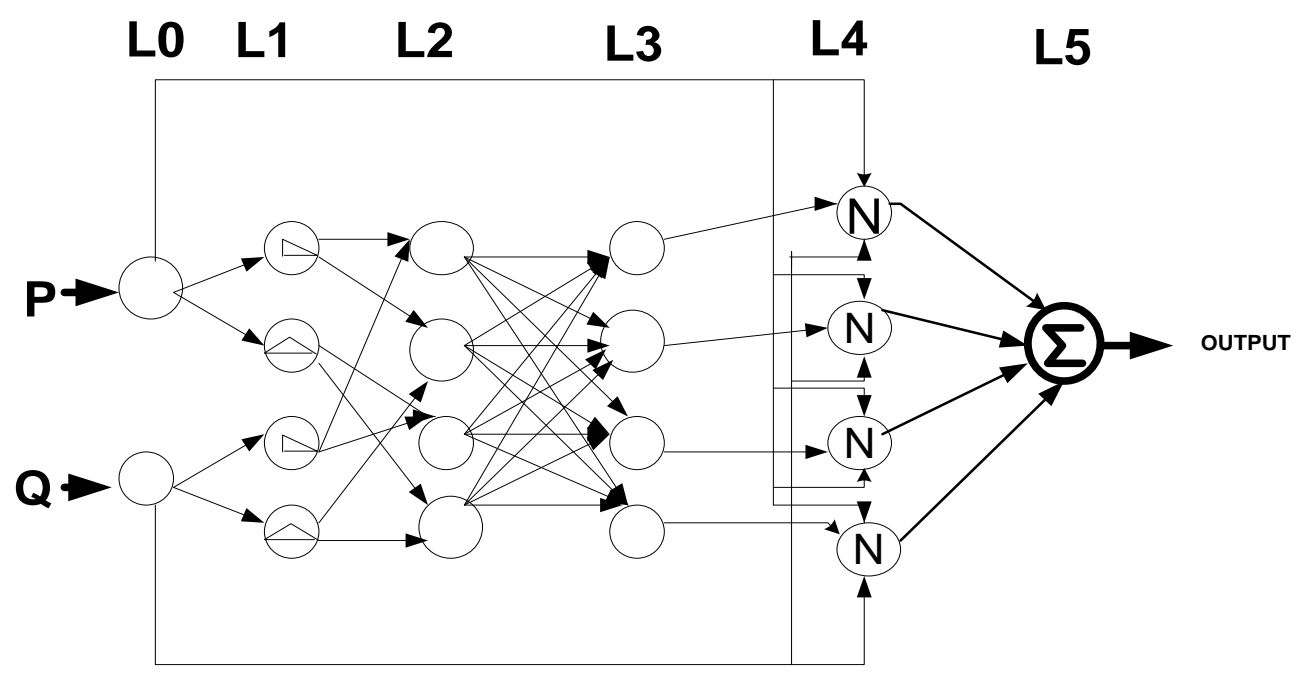

Fig. 1. Construction of an ANFIS

ANFIS technique is based upon data processing. It produces a felicitous input/output mapping with membership function which is based upon fuzzy IF-THEN rules to procreate the input output pairs. These control parameters can be adjusted by using back propagation technique [3]. Therefore the I/O data must be operated on a large range so that the control performance of ANFIS will be guaranteed otherwise the previous designed controller will not yield the precise output or result.

In the above five layer structure shown in fig.1, the first and fourth layer's node is adaptive and other nodes are fixed. To optimize the approaching performance, the adaptive nodes parameters can be changed by one or more learning algorithms. It uses the feed forward, back propagation network, which has a simplified method for the learning process that comprises of providing the data having patterns of inputs and the target outputs, accessing the network performance, adaption of connection strength to produce better output.

Three basic stages comprise of fuzzy controllers, commonly known as fuzzification, fuzzy interface and defuzzification. The above figure shows the different layers of the back propagation interface. In neural network the I/O behavior of the system can be determined by the use of weights between the input and output layers. The parameters obtained in the fuzzification and defuzzification process of the fuzzy logic system can thus be trained using the updated weights of a neural network [7].Design parameters of an adaptive neuro-fuzzy inference system controller are a crucial part to obtain a useful and valuable output. Hence its design process can be divided into two steps [8].

The hybrid approach combined with fuzzy logic and artificial neural network is considered in many applications successfully and also few works have been done on fault classification purpose of the power system. Looking to the extensive use and higher performance, in this work the Neuro-Fuzzy (NF) classifier also considered as one of the approach. The neural network has the edge over other techniques in terms of robustness, work efficiently with both quantitative and qualitative data, computational burden and don't need exact mathematical modeling of the system $[9,10]$. Generally, the classification process through NF approach follows two major steps during implementation as a fault classifier. It produces the fuzzy sets in the first step by collecting the data in the form of voltage measurement at PCC, and then fuzzifying the data to apply different fuzzy rules and defuzzification to get the desired output. Fuzzy rule base with IF-THEN rules and appropriate membership functions are considered judiciously because these are problem dependent. In this approach, ANFIS combines the parallel computing, learning capability and knowledge representation of neural networks in the form of interpretation of learning ability in fuzzy systems. Neural networks are therefore becoming more transparent and the system is able to understand, integrating both symbolic and numerical knowledge of the 
mechanism in the NF model. The automatic linguistic rule extracts one of the important features of NF when there is no information or very less information about the data set relevant to any signal.

An NF model of a non-linear dynamic system can be implemented from empirical evidence. Insight information about the dynamical and linearity properties of the system can be assessed by NF model as a classifier. Because of its simple rule base design and application to uncertain and complex system, NF models are used in many industrial process applications such as the financial system, and many others. Whereas, better performance of the NF method as a classifier depends on the knowledge base or the rule base and system parameters about the empirical data and system.

\section{A. Constructing Step}

In this step the type and number of I/O membership function is evaluated. Membership function comprises of several types such as Gaussian, triangular etc. The membership function can be chosen as 3,5 and so on. By choosing the suitable number of input membership function the number of rules can be decreased which leads to increase in learning speed. But the linear output membership function has to be a constant value.

\section{B. Training Step}

Training step followed by the above step a learning algorithm and training parameters are chosen. ANFIS uses back propagation algorithm to obtain these parameters. The network output is compared with the desired output in this process and the resulting error is used to adjust the weights between the layers in order to reduce the error. After repeated training process it is expected that the final error would reduce to an acceptable output value.

The whole control scheme for the classification of fault of the system is show in figure a [4]. These modules comprise the whole system. The sample data is processed and through the sequence module, different fault type will be judged. With the help of the phase modules, the tripping signal will be provided for the specific type of fault.

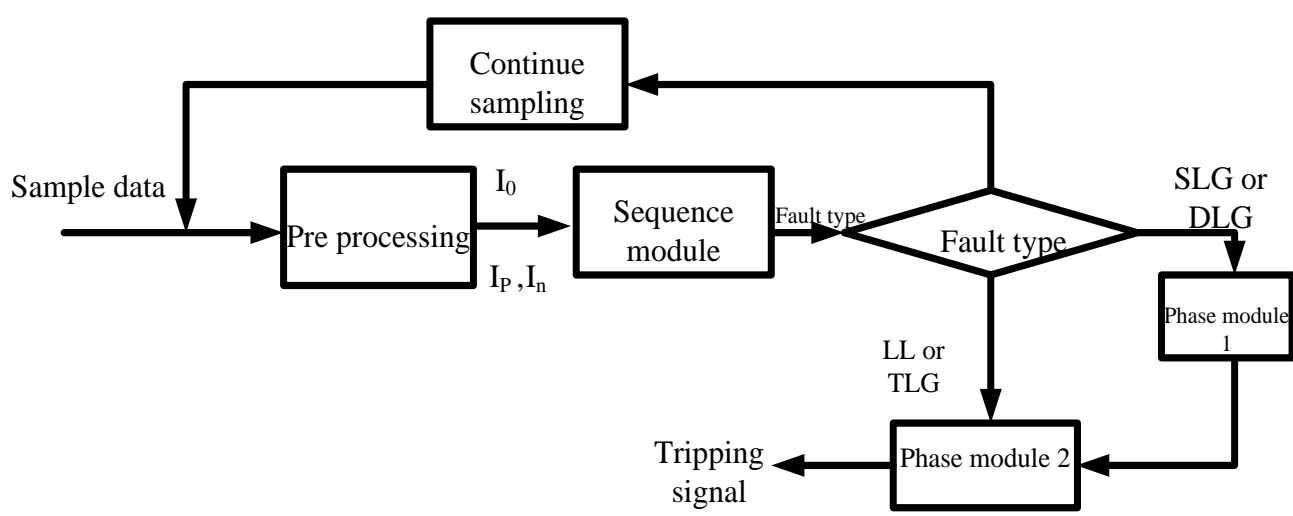

Fig. 2. Control scheme of ANFIS

These models are executed via a structured computer software package which helps with interactive debugging to update the structure and parameters. During the training process the entire process can be managed directly. The model can be trained by using information from various sources, the learning of designers and sample data. With the help of the independent set of test patterns the neuro fuzzy fault classifier was tested. The main objective of the testing is to achieve speed, reliability and the generalisation of the classifier of neuro fuzzy fault.

\section{Proposed Model}

A simulation model has been brought up to carry out the research on the operation and behaviour of power system, in the presence of distributed generation under distinct cases. The proposed simulated model is supposed to exhibit a reliable system with all its indispensable components. In the distribution level diesel generator is connected along with micro turbine and doubly fed induction generator wind turbine. The single line diagram of proposed model is shown in figure 3, which contains a three phase source, three phase transformer, circuit breaker, transmission line, three phase load and a DG set at the load end. 


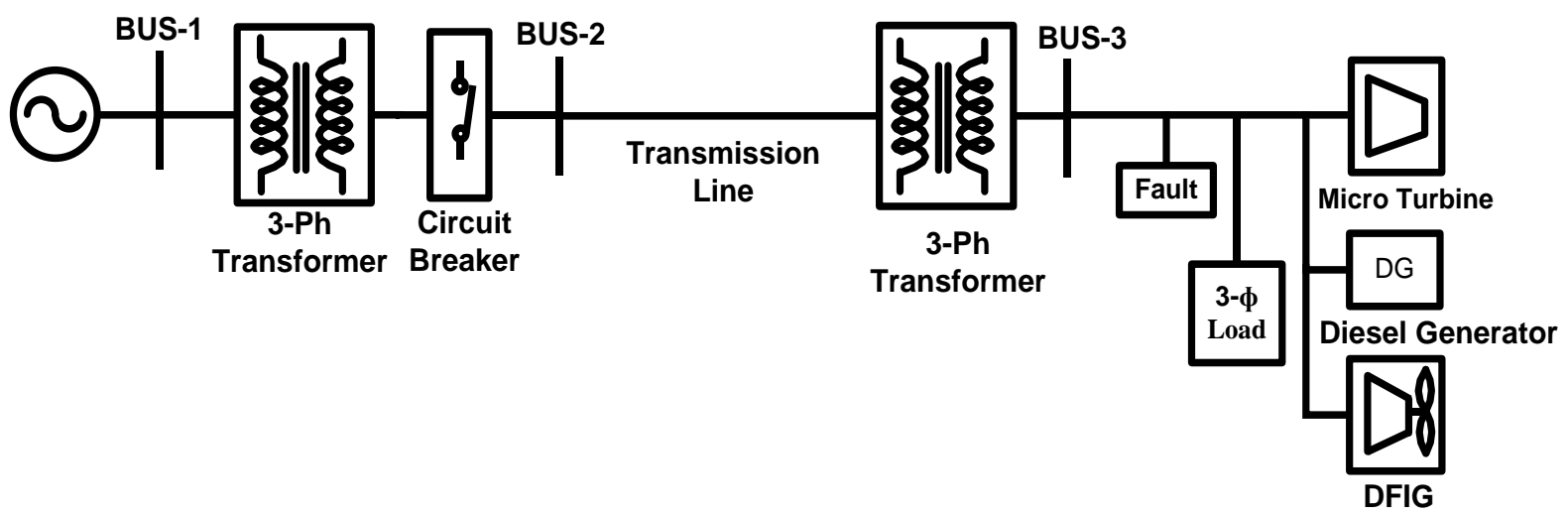

Wind Turbine

Fig. 3. Single line diagram of micro grid

At the source end, the step up transformer is used to minimise the transmission losses by raising the source voltage and the step down transformer is used at the distribution level to lower the voltage to a normal distribution level. Both the transformers are connected in between a pi section transmission line of $60 \mathrm{~km}$. A circuit breaker is connected in series with the line at beginning of transmission which behaves as a normally closed switch under healthy condition. Voltage signals are separated from the typical coupling point, which is at the level of distribution. A fault block is also connected at the distribution level which is connected to the point of common coupling.

\section{Simulation and Results}

If the input dimension space is low, then this particular method is fast and implementation is also easy to use. As it uses the error back-propagation, so more chances are there that it can loss transparency and local model accuracy. Error back-propagation is not a local nonlinear optimization procedure; it is following global optimization procedure. Subset of input spaces is used for local rules, but it is activated by higher level rules. Based on the fault disturbances and the system characteristics, NF model is designed. NF model can pre-process the data and then it detects the disturbance and classification of fault disturbance and islanding. A NF system is a neural network and operates in a fuzzy mode of inference. Developing training structures according to the IFTHEN rules and feature decisions can be input and output variables.

Training data used to train the fault classification data are taken at a fault distance of $20 \mathrm{~km}, 30 \mathrm{~km}, 40 \mathrm{~km}$, and $50 \mathrm{~km}$ for various forms of fault and insulation (i.e. single phase to ground, phase to phase, double phase to ground or tri-phase fault) and with different fault resistance $(0.001,0.01,0.1,10,100 \mathrm{ohm})$. The performance measurement using NF at various epochs is shown in Figure 4.

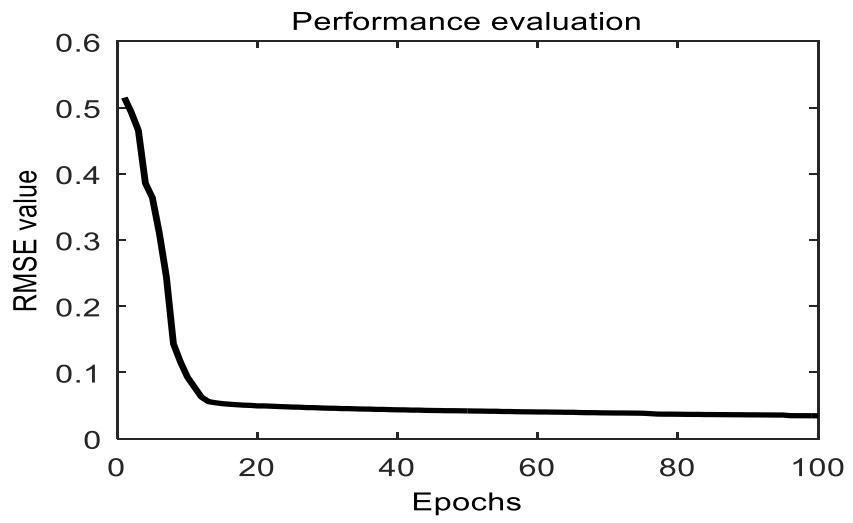

Figure 4. Error value at different epochs (NF)

The data is derived from the values of the voltage measurements. The voltage calculation accumulates 126 characteristics data, which offers 63 training data and is left over for research. Half of all the data on characteristics is taken for review. The exact percentage of the fault and islanding classification in a micro grid using Neuro fuzzy in this technique is 92.06 percent. 


\section{Conclusion}

The classification of faults using Neuro Fuzzy (NF) has been suggested in this paper. Test results show that the training of the neural network classifier can render rapid and accurate classification in various systems and conditions of fault. The neuro-fuzzy research and training data was gathered from different fault and islanding environments. The proposed method of fault classification using Neuro Fuzzy contributed correct classification nearly about $92 \%$ for distinct abnormalities and islanding with different set of resistance. The results of the simulation show that this method is very specific and stable under tested operating conditions, including cases where noisy situations are introduced into the device or signals.

\section{References}

1. Dalstein, T., \& Kulicke, B. (1995). Neural network approach to fault classification for high speed protective relaying. IEEE Transactions on Power Delivery, 10(2), 1002-1011.

2. Nanda, R.P., Bisoi, D., Behera, A., Panigrahi, B.K., \& Satapathy, A.K. (2019). Classification of Faults in a Wind Connected Power System Using Artificial Neural Network. In 2019 3rd International Conference on Computing Methodologies and Communication (ICCMC), 504-507.

3. Jang, J. S. (1993). ANFIS: adaptive-network-based fuzzy inference system. IEEE transactions on systems, man, and cybernetics, 23(3), 665-685.

4. Kusagur, A., Kodad, S.F., \& Ram, B.S. (2010). Modeling, design \& simulation of an adaptive neurofuzzy inference system (ANFIS) for speed control of induction motor. International Journal of Computer Applications, 6(12), 29-44.

5. Salam, A.A., Mohamed, A., \& Hannan, M.A. (2008). Technical challenges on microgrids. ARPN Journal of engineering and applied sciences, 3(6), 64-69.

6. Dubois, D., \& Prade, H. (1998). An introduction to fuzzy systems. Clinica Chimica Acta, 270(1), 3-29.

7. Padhy, S.K., Panigrahi, B.K., Ray, P.K., Satpathy, A.K., Nanda, R.P., \& Nayak, A. (2018). Classification of faults in a transmission line using artificial neural network. In 2018 International conference on information technology (ICIT), 239-243.

8. Kusagur, A., Kodad, S.F., \& Ram, B.S. (2010). Modeling, design \& simulation of an adaptive neurofuzzy inference system (ANFIS) for speed control of induction motor. International Journal of Computer Applications, 6(12), 29-44.

9. Panigrahi, B. K., Ray, P. K., Rout, P. K., Mohanty, A., \& Pal, K. (2018). Detection and classification of faults in a microgrid using wavelet neural network. Journal of Information and Optimization Sciences, 39(1), 327-335. https://doi.org/10.1080/02522667.2017.1374738

10. Panigrahi, B.K., Ray, P.K., Rout, P.K., \& Sahu, S.K. (2017). Detection and location of fault in a micro grid using wavelet transform. In 2017 International Conference on Circuit, Power and Computing Technologies (ICCPCT), 1-5. 